\title{
Diagrams in Essays: Exploring the Kinds of Diagrams Students Generate and How Well They Work
}

\author{
Emmanuel Manalo $^{1(\bowtie)}$ and Mari Fukuda ${ }^{2}$ \\ ${ }^{1}$ Graduate School of Education, Kyoto University, Kyoto, Japan \\ manalo.emmanuel.3z@kyoto-u.ac.jp \\ ${ }^{2}$ Graduate School of Education, The University of Tokyo, Tokyo, Japan \\ mari_fukuda@p.u-tokyo.ac.jp
}

\begin{abstract}
Using appropriate diagrams is generally considered efficacious in communication. However, although diagrams are extensively used in printed and digital media, people in general rarely construct diagrams to use in common everyday communication. Furthermore, instruction on diagram use for communicative purposes is uncommon in formal education and, when students are required to communicate what they have learned, the usual expectation is they will use words - not diagrams. Requiring diagram inclusion in essays, for example, would be almost unheard of. Consequently, current understanding about student capabilities in this area is very limited. The aim of this study therefore was to contribute to addressing this gap: it comprised a qualitative exploration of 12 undergraduate students' diagram use in two essays (in which they were asked to include at least one diagram). Analysis focused on identifying the kinds of diagrams produced, and the effectiveness with which those diagrams were used. Useful functions that the diagrams served included clarification, summarization, integration of points, and provision of additional information and/or perspectives in visual form. However, there were also redundancies, as well as unclear, schematically erroneous, and overly complicated representations in some of the diagrams that the students constructed. These findings are discussed in terms of needs, opportunities, and challenges in instructional provision.
\end{abstract}

Keywords: Self-constructed diagrams $\cdot$ Essay writing $\cdot$ Effective communication $\cdot$ Student instructional needs

\section{Introduction}

Alongside problem solving and thinking, communication is one of the areas of human activity where diagram use is considered to be beneficial. When appropriately used, diagrams can clarify and/or complement verbal information presented in speech or text, so that both verbal and visual channels of working memory are utilized, thus facilitating more efficient cognitive processing $[1,2]$.

Diagrams can contribute to both message encoding and decoding (i.e., the production and the comprehension of communication), thus being of value to both the communicator and the communication receiver. Especially in contexts where there are 
some constraints or limitations to conveying the message through verbal means, diagrams can be indispensable. They can supplement speech or text by providing complementary or alternative means of conveying the intended message. Examples of such contexts include communicating complicated procedures, like furniture assembly [3], and communicating with people who speak a different language [4].

However, despite the apparent usefulness of diagrams in communication, its actual use remains very limited. Pictures and various kinds of diagrams, including illustrations, are regularly used in books, magazines, websites, and various forms of printed and digital media, but most of those visual representations are commercially or professionally created. They are not generated by regular people in everyday communication contexts. Regular people are often only receivers of such visual representations. In most communication contexts, they do not generate their own diagrams: they rely almost exclusively on written or spoken words. In formal education provided in modern societies, diagram use for communicative purposes is rarely taught. Despite the recognition in research and policy documents of the value of being able to use multiple forms of representation [5, 6], students seldom receive explicit instruction about how to create and use diagrams. In both school and higher education, when students are asked to communicate what they have learned and what they think (e.g., in essays, which are focused pieces of writing intended to inform or persuade), the general expectation is that they will express that information in words - without the use of any diagrams [7].

Considering that much of the knowledge and ideas that students have to engage with, learn, and then communicate are quite complex, and diagrams have the capacity of representing complex ideas effectively [8], the general lack of attention in education to cultivating skills in diagram use is troubling. Like words, diagrams can be used effectively or ineffectively [9], so the question of the extent to which instruction or guidance may be necessary would appear important to address. In tasks like problem solving and information organization in subjects like mathematics and science, the kinds of diagrams that students generate and use have previously been investigated [10-12]. However, very few studies have examined diagram use in communicating information in the social sciences [13], where traditionally a greater emphasis has been placed on the quality of language that is used. In fact, the present authors are not aware of any studies that have examined students' diagram use in essays. Our current understanding of student capabilities in using diagrams in such contexts is very limited, including what we know of the potential benefits that such use might afford.

The present study was motivated by this knowledge gap, and it comprised a qualitative exploration of student diagram use in two essays they produced for an undergraduate-level introductory course in educational psychology. In the two essays, the students were asked to include at least one diagram to portray processes or mechanisms of moderately complex ideas. Both essays were expository-type essays, hence requiring the students to demonstrate not only knowledge of the topic, but also the ability to communicate information clearly - which the appropriate use of diagrams is supposed to facilitate. The following were the main questions we addressed:

(i) What kinds of diagrams would students use to portray processes/mechanisms?

(ii) In what ways do students use diagrams effectively in their essays?

(iii) In what ways do they not use diagrams effectively? 


\section{Method}

This investigation comprised analysis of the contents of two essays that students produced as part of their coursework. No experimental manipulation was involved. The analysis was conducted following completion of the course, so it had no bearing on the students' grading. Permission was obtained from all the students for use of their essays.

There were 12 students in the course (females $=4$ ), 7 of whom were in their first year of study, while the remaining 5 were in their second year or higher. Nine of the students were Japanese, and 3 were international students from other East Asian countries. All had English as a foreign language, but were adequately proficient in that language (a requirement for acceptance to the university). The course was conducted entirely in English, and all assignments (including the essays) had to be written in English.

The essays were each worth $20 \%$ of the students' final grade, and they dealt with topics covered in the course. However, both essays required students to seek additional information (beyond what was covered in class), and to provide explanations that could not simply be obtained from the instructions provided in the course. The first essay required the students to research and then explain one theory about how young children develop their understanding of the world around them. The second required them to undertake research on formative assessment and explain how it can promote more successful learning. In both essays, the students were asked to include at least one diagram, which was allotted 3 points (out of 20) in the grading rubrics (in this case, for "demonstrating a clear understanding of the mechanisms or processes that it illustrates"). Diagrams can assist in clarifying ideas [8] and so, from a pedagogical perspective, one purpose of asking the students to include the diagram was for them to better understand key processes covered in the course. The diagram had to be self-constructed (i.e., not copied-and-pasted from some other source). In the first essay, the instruction given to the students indicated that the diagram was to "help in explaining the progress in understanding that children develop", while in the second essay the diagram was to "help in clarifying how formative assessment facilitates learning". No other instruction was provided on what form the diagram should take or how they should construct it, and no diagrams relating to those or other similar mechanisms/processes were shown during instructions provided in class. During grading of the essays, apart from the score out of 3 on the grading rubrics (see above), no explicit comment or feedback was provided on the type, content, or quality of construction of the diagrams the students included.

In the analysis, firstly the number and kinds of diagrams included in the essays were determined with the use of a coding schema comprising categories from previous research $[10,11,13]$. Apart from the first author's coding, the second author, who initially was not involved in this research, also independently coded the diagrams. Initial inter-coder agreement was $75 \%$. Differences were then discussed and subsequently agreed upon. Second, the diagrams were evaluated in terms of how effectively they were used. For this, key questions asked were: Does the diagram contribute to clarifying the process it refers to - and, if so, how? Apart from clarification, does it serve other useful functions? When diagrams did not appear to work well, the reasons were also carefully considered. Again, both authors independently coded the diagrams (initial inter-coder agreement was $92 \%$ ), and then discussed differences to reach agreement. 


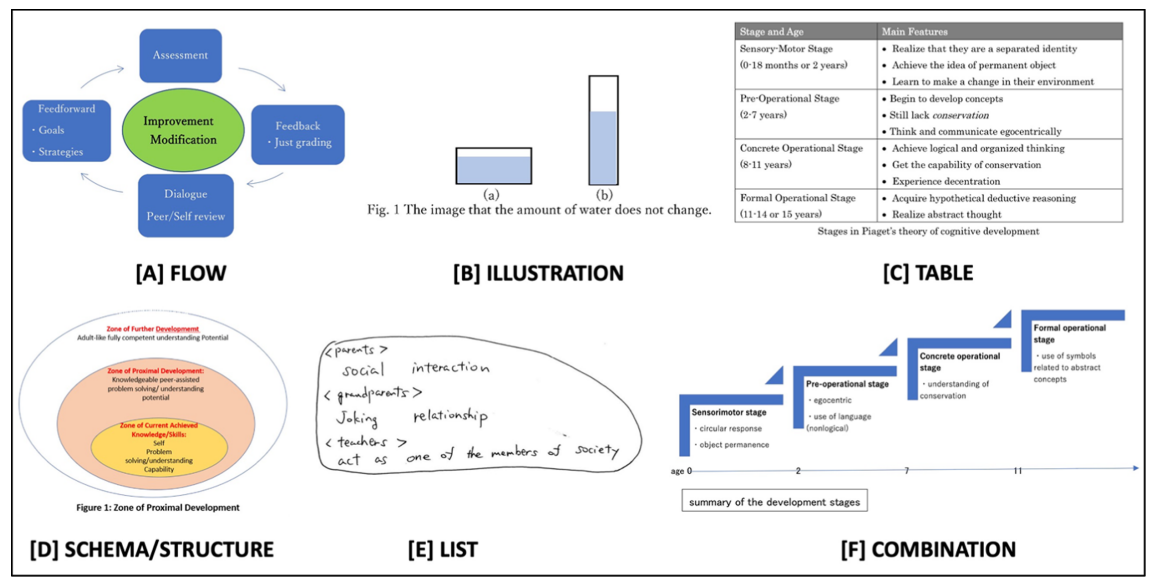

Fig. 1. Examples of diagrams belonging to each of the categories that were identified

\section{Results and Discussion}

\subsection{Kinds of Diagrams Used to Portray Processes/Mechanisms}

Table 1 shows the kinds/categories and corresponding frequencies of diagrams the students included in their first and second essays, and in total, while Fig. 1 shows examples of the diagrams belonging to each of those categories. In the first essay four of the 12 students included more than one diagram (two student with 3 diagrams, and two with 2 diagrams), and in the second essay two students included more than one diagram (both with 2 diagrams). The frequencies shown in Table 1 include all the diagrams the students generated.

All except one student included selfconstructed diagrams (as the assignments required). We were fairly confident about this because the language use in and appearance of the diagrams included suggested non-native and/or non-professional creators. The one student who included diagrams that obviously came from some Internet source (they were both watermarked) did so in both the first and second

Table 1. Kinds of diagrams and the frequencies with which they were used

\begin{tabular}{l|l|l|l}
\hline Kinds of diagram & Essay 1 & Essay 2 & Total \\
\hline Flow & 3 & 7 & 10 \\
\hline Illustration & 5 & 2 & 7 \\
\hline Table & 1 & 2 & 3 \\
\hline Schema/Structure & 2 & 0 & 2 \\
\hline List & 1 & 0 & 1 \\
\hline Combinations & 6 & 3 & 9 \\
\hline
\end{tabular}
of illustrations.

Although a "list" on its own does not - technically speaking - count as a diagram, it has been included in the categories because one student erroneously included a list as one of his 3 diagrams for the first essay, and three other students included lists as part of their "combination" diagram. 
The kind of diagram most frequently used was a flow diagram: apart from the total of 10 flow diagrams shown in Table 1, 7 of the 9 combination diagrams comprised a flow diagram with another kind of diagram. This is probably understandable given that flow diagrams (also known as "flow charts") are meant to depict processes, procedures or sequence of steps, and cause-and-effect relationships. An interesting point to note is how the number of flow diagrams increased from the first to the second essay. No instruction or hint was given to the students about what diagram to use, so this increase could have been due to a number of other possible reasons, including differences between the two essays in the procedures/mechanisms that needed to be represented, the students seeing other diagrams their peers have generated (although there were no indications of copying), and development in the students' understanding of what works well (or not) in using diagrams to communicate particular kinds of information.

It is also worth noting that although we often consider flow diagrams as being most appropriate for representing processes and mechanisms, other forms of diagrams can work as effectively when designed well to match their intended purposes. For example, Panels $\mathrm{C}$ and $\mathrm{F}$ in Fig. 1 show two examples depicting the progression through the stages of Piaget's theory of cognitive development, using a table in $\mathrm{C}$, and a combination of illustration (of steps) and line diagram in F. Although they differ in appearance, the diagrams can be considered as working equally well not only in showing the proposed stages of the theory, but also in conveying the incremental progression through key cognitive abilities with increasing age (corresponding to those stages).

\subsection{Ways that Diagrams Were Used Effectively}

The majority of the students did not refer to their diagrams in the text of their essays: only two students did in both essays. Thus, this is perhaps an academic writing method that undergraduate students (like these students) could usefully be instructed to do. However, in general, the students placed their diagrams appropriately, following the text where they deal with the information that is portrayed in the diagram - thereby making the connection between text information and the diagram more apparent.

Concerning the question of whether the diagrams that the students constructed contributed to clarifying any of the processes or mechanisms they were explaining: in the first essay, 7 of the students were considered to have satisfactorily achieved this with at least one of their diagrams, while 8 of them were considered to have done so in the second essay. In each of these cases, the diagrams served a useful function in the essay, to the extent that if they were not included, something sufficiently important in the essay would have been lost, not achieved, or not conveyed as adequately. In most of the cases, the diagrams clarified how the stages or processes referred to in the essay text connect or relate to each other and progress through particular sequences: Panels A and D in Fig. 1 are good examples of this. However, in a few cases, the diagrams also made clearer concepts that - to those unfamiliar with them - could be difficult to understand, such as what is involved in developing the ability of conservation (Panel B of Fig. 1).

In some cases, the diagrams also showed or clarified the connections to other components, such as children's abilities in connection to the progression of developmental stages in the previously referred to Panels C and F in Fig. 1. In a way, some of 
the diagrams that worked well served a summarizing function: they visually represented key components and showed more saliently how they were related to each other - which were not as easy to apprehend in sentences because of temporal/sequential separation. This is one of the reasons diagrams are considered effective: they integrate all information that is used together, reducing the need and effort for searching [14].

In the majority of cases where the diagrams worked well, they visually represented content that was already represented in words in the text - albeit with some enhancements like integration, as noted above. However, in a few exceptional cases, the diagrams also introduced content that was not present in the text of the essays. Figure 2 provides two examples of this. In Panel A, the diagram includes details in the lower part about unsuccessful (arrows with $\mathrm{x}$ ) and successful (arrows with o) outcomes which require different responses. These details were not explicitly provided in the text but they enable readers to better understand how formative feedback is used in the example of solving story problems. Likewise, in Panel B, the diagram shows details not duplicated in the text about how different categories of complexes are formed, leading eventually to the formation of concepts [15]. The illustrations of different object combinations make the categories of complexes easier to grasp and distinguish from each other.

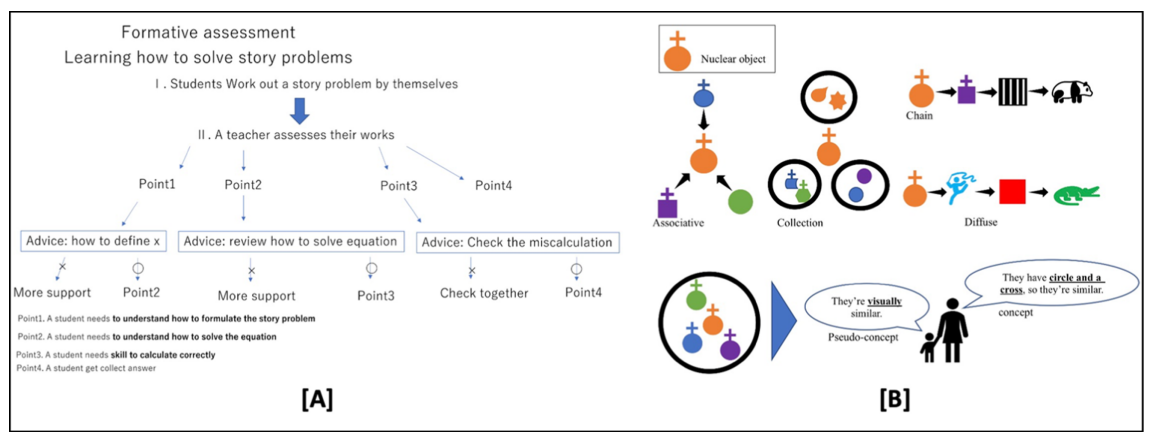

Fig. 2. Examples of diagrams that introduced content or elaborations not present in the text

\subsection{Ways that Diagrams Were Not Used Effectively}

There were also numerous instances when the diagrams the students included did not appear to serve any useful function in the essay. In a couple of those cases, the diagrams were redundant: they showed images that portrayed information from the text that was simple enough not to require visual clarification. The illustration in Panel A in Fig. 3 is an example of this. Another ineffective use manifested was when the schematic structure of the diagram was unclear or erroneous. Examples of this are shown in Panels B and C of Fig. 3. In Panel B, both the intended message and the connections between the components shown are unclear. In Panel $\mathrm{C}$, the meaning of the arrows, and therefore what process might be depicted by the diagram, is unclear. In addition, there 
were a few diagrams, like the one shown in Panel D of Fig. 3, which were quite complicated and therefore hard to understand. The contents of Panel D were also referred to in the text of the student's essay, but the relationships shown in the diagram are new configurations that are not obvious and not explained explicitly in the text. It is therefore difficult to grasp its possible contribution to explaining, in this case, children's development of understanding of the world around them.

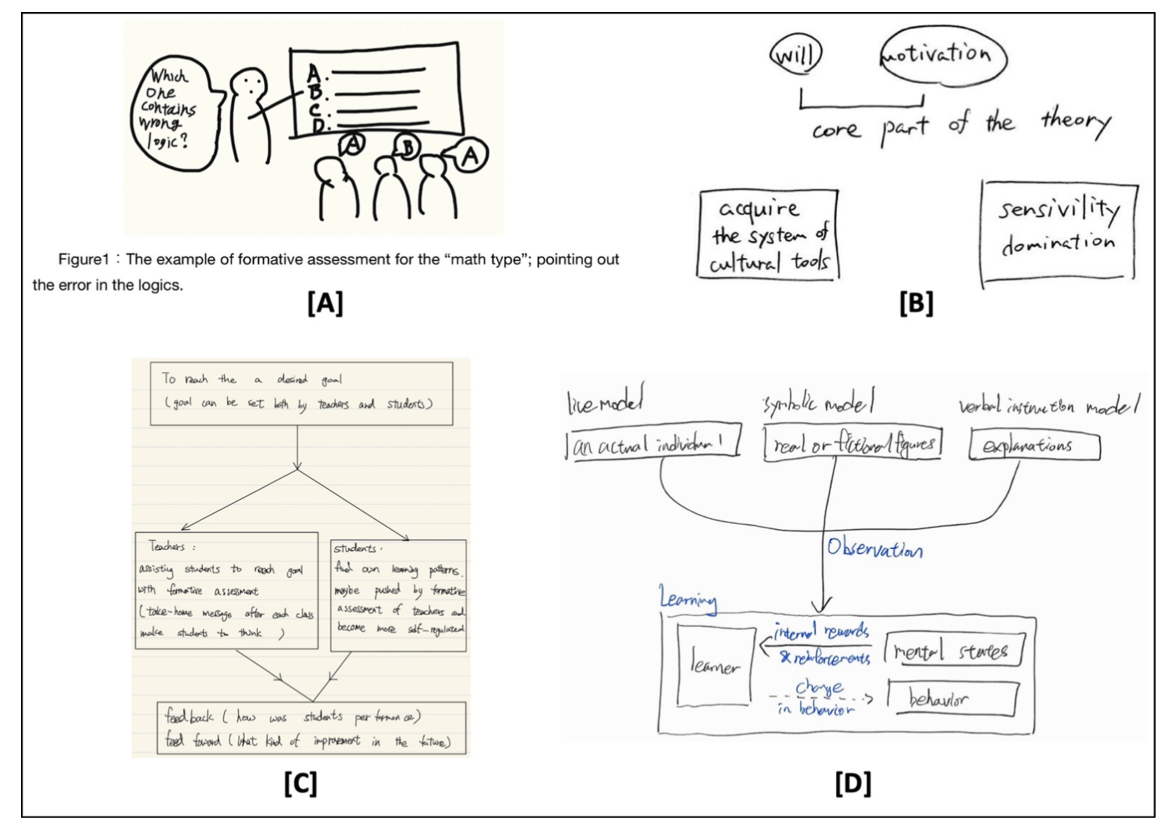

Fig. 3. Examples of diagrams that did not effectively serve their intended purpose in the essay

\subsection{Implications for Theory, Research, and Practice}

The many purposes that diagrams can serve in enhancing communication have been established in previous research [2, 5, 8], and the findings of the present study provide additional evidence for those in the area of student essay writing at the tertiary level. The findings also provide support for the idea that the same communicative purpose can be served by different kinds of diagrams [14]. Thus, for example, conveying the progression of a particular process can effectively be achieved using a flow diagram or a table ... or an illustration. This means that, in the same way that different words can convey the same meaning, different diagrams - if used/constructed appropriately can communicate the same meaning. However, in future research, it would be useful to examine the range of communicative purposes that different kinds of diagrams can serve as there are probably important limitations to it. For example, if the communicative purpose is to describe what something looks like, other kinds of diagrams may 
not be quite as effective as an illustration (e.g., a table or a flow chart would be quite limited in conveying qualities pertaining to appearance).

The findings of the present research also suggest that many students, even at the tertiary level, would likely benefit from receiving some instruction or guidance on the use of diagrams not only in essays but also other forms of communication. While some students were able to generate diagrams that served useful functions in their essays, there were also quite a few who did not manage to do this. However, the findings of this exploratory study were based on a small sample of students taking the same course, so future investigations about spontaneous use and the effects of instruction provision ought to be conducted with larger and more diverse student groups.

Acknowledgment. This research was supported by a grant-in-aid (20K20516) received from the Japan Society for the Promotion of Science.

\section{References}

1. Clark, J.M., Paivio, A.: Dual coding theory and education. Educ. Psychol. Rev. 3, 149-210 (1991)

2. Mayer, R.E., Moreno, R.: A split-attention effect in multimedia learning: evidence for dual processing systems in working memory. J. Educ. Psychol. 90, 312-320 (1998)

3. Heiser, J., Tversky, B.: Characterizing diagrams produced by individuals and dyads. In: Freksa, C., Knauff, M., Krieg-Brückner, B., Nebel, B., Barkowsky, T. (eds.) Spatial Cognition 2004. LNCS (LNAI), vol. 3343, pp. 214-226. Springer, Heidelberg (2005). https://doi.org/10.1007/978-3-540-32255-9_13

4. Alabsi, T.A., Taha, I.M.: Using drawings to facilitate communication of non Arabic and non English speaking patients in Al Medinah health care sector. J. Am. Sci. 10(6), 174-190 (2014)

5. Ainsworth, S., Prain, V., Tytler, R.: Drawing to learn in science. Science 333, 1096-1097 (2011)

6. National Research Council: Education for Life and Work: Developing Transferable Knowledge and Skills in the 21st Century. National Academies Press, Washington, DC (2012)

7. Manalo, E., Ueaska, Y., Kriz, S., Kato, M., Fukaya, T.: Science and engineering students' use of diagrams during note taking versus explanation. Educ. Stud. 39, 118-123 (2013)

8. Tversky, B.: Visualizing thought. Top. Cogn. Sci. 3, 499-535 (2011)

9. Hegarty, M., Kozhevnikov, M.: Types of visual-spatial representations and mathematical problem solving. J. Educ. Psychol. 91, 684-689 (1999)

10. Novick, L.R., Hurley, S.M.: To matrix, network, or hierarchy: that is the question. Cognitive Psychol. 42, 158-216 (2001)

11. Zahner, D., Corter, J.E.: The process of probability problem solving: use of external visual representations. Math. Think. Learn. 12, 177-204 (2010)

12. Manalo, E., Uesaka, Y.: Students' spontaneous use of diagrams in written communication: understanding variations according to purpose and cognitive cost entailed. In: Dwyer, T., Purchase, H., Delaney, A. (eds.) Diagrams 2014. LNCS (LNAI), vol. 8578, pp. 78-92. Springer, Heidelberg (2014). https://doi.org/10.1007/978-3-662-44043-8_13 
13. Manalo, E., Uesaka, Y.: Hint, instruction, and practice: the necessary components for promoting spontaneous diagram use in students' written work? In: Jamnik, M., Uesaka, Y., Elzer Schwartz, S. (eds.) Diagrams 2016. LNCS (LNAI), vol. 9781, pp. 157-171. Springer, Cham (2016). https://doi.org/10.1007/978-3-319-42333-3_12

14. Larkin, J.H., Simon, H.A.: Why a diagram is (sometimes) worth ten thousand words. Cognitive Sci. 11, 65-99 (1987)

15. Vygotsky, L.: Studies in communication. In: Hanfmann, E., Vakar, G. (eds.) Thought and Language. MIT Press, Cambridge (1962)

Open Access This chapter is licensed under the terms of the Creative Commons Attribution 4.0 International License (http://creativecommons.org/licenses/by/4.0/), which permits use, sharing, adaptation, distribution and reproduction in any medium or format, as long as you give appropriate credit to the original author(s) and the source, provide a link to the Creative Commons license and indicate if changes were made.

The images or other third party material in this chapter are included in the chapter's Creative Commons license, unless indicated otherwise in a credit line to the material. If material is not included in the chapter's Creative Commons license and your intended use is not permitted by statutory regulation or exceeds the permitted use, you will need to obtain permission directly from the copyright holder.

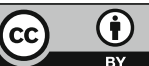

